\title{
IN-SITU FABRICATION OF COMPOSITE PIEZOELECTRIC WAFER ACTIVE SENSORS FOR STRUCTURAL HEALTH MONITORING
}

\author{
Victor Giurgiutiu, PhD, PE, Member ASME \\ Department of Mechanical Engineering \\ University of South Carolina \\ Columbia, SC 29208 \\ 803.777.8018, victorg@sc.edu
}

\author{
Bin Lin \\ Department of Mechanical Engineering \\ University of South Carolina \\ Columbia, SC 29208 \\ linbin@engr.sc.edu
}

\begin{abstract}
Structural health monitoring (SHM) is important for reducing maintenance costs while increasing safety and reliability. Traditionally, structural integrity tests required attachment of sensors to the material surface. This is often a burdensome and time-consuming task, especially considering the size and magnitude of the surfaces measured (such as aircraft, bridges, structural supports, etc.). Temporary sensors are a hassle to install; there are some critical applications where they simply cannot accomplish the task required. Piezoelectric wafer active sensors (PWAS) can be permanently attached to the structure and offer a permanent sensor solution. Existing ceramic PWAS, while fairly accurate when attached correctly to the substance, may not provide the long term durability required for SHM. The bonded interface between the PWAS and the structure is often the durability weak link. Better durability may be obtained from a built-in sensor that is incorporated into the material.

This paper describes the work on the in-situ fabrication of PWAS using a piezoelectric composite approach. The piezoelectric composite was prepared by mixing small lead zirconate titanate (PZT) particles in an epoxy resin matrix; the mixture was then directly applied onto the surface of a host structure using a designed mask. The curing of the piezo composite was carried out at elevated temperature. After curing, the cured composite was
\end{abstract}

sanded down to the desired thickness. Finally, the piezo composite was poled under a high electric field to activate the piezoelectric effect. The resulting insitu composite PWAS was utilized as a sensor for dynamic vibration and impact. Characterization of the in-situ composite PWAS on aluminum structure have been recorded and compared with ceramic PWAS before and after poling. To evaluate the performance of the in-situ composite PWAS, both vibration and impact tests were conducted. In-situ composite PWAS are believed to be a good candidate for reliable low-cost sensor fabrication for SHM.

\section{INTRODUCTION}

Structural health monitoring (SHM) addresses an urgent need of our aging infrastructure. In recent years, several investigators (Keilers and Chang ${ }^{[1]}$, Chang ${ }^{[2][3]}$, Wang and Chang ${ }^{[4]}$, Lin and Yuan ${ }^{[5][6]}$, Ihn and Chang ${ }^{[7]}$, Giurgiutiu et al ${ }^{[8][9]}$, and others) have explored the generation and detection of structural waves with piezoelectric wafer active sensors (PWAS). Most of the methods used in conventional NDE, such as pitch-catch, pulse-echo, and phased arrays, have also been demonstrated experimentally with PWAS (Giurgiutiu et al. ${ }^{[9][10]}$ ). These successful experiments have positioned PWAS as an enabling technology for the development and implementation of active SHM systems. PWAS are inexpensive, non-intrusive, un-obtrusive, and minimally invasive. They can be surface-mounted on 
existing structures, inserted between the layers of lap joints, or placed inside composite materials. Figure 1 shows an array of $7 \mathrm{~mm}$ square PWAS mounted on an aircraft panel, adjacent to rivet heads and an electric discharge machined (EDM) simulated crack. The minimally invasive nature of the PWAS devices is apparent. A PWAS weighs $0.068 \mathrm{~g}$, is $0.2 \mathrm{~mm}$ thick, and costs around $\$ 7$ each. In contrast, a conventional ultrasonic transducer weights $50 \mathrm{~g}$, are $20 \mathrm{~mm}$ thick, and cost around \$300.PWAS are used in SHM applications are able to detect structural damage using Lamb waves. PWAS are small, lightweight, unobtrusive, and low cost PWAS achieve direct transduction between electric and elastic wave energies. PWAS are essential elements in the Lamb-wave SHM with pitch-catch, pulse-echo, and electromechanical impedance methods.

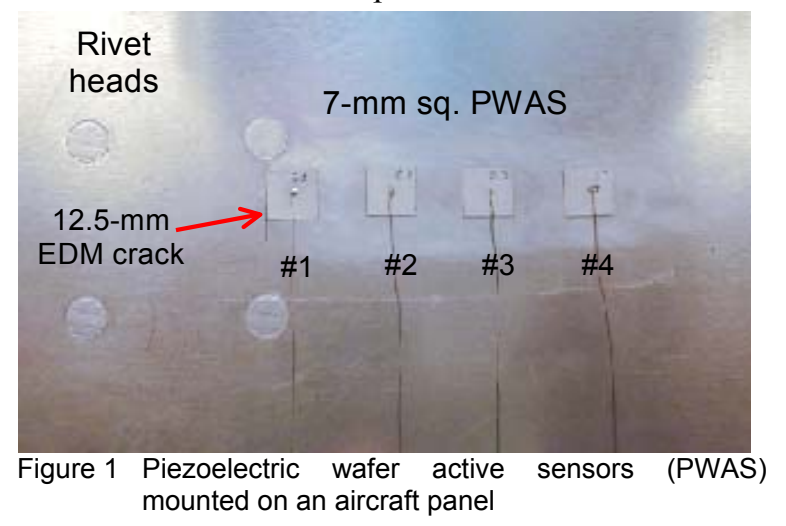

However, there is something that needs to be addressed:

(1) Current PWAS are adhesively bonded to the substrate structure (Figure 2a) or incorporated between the layers of a laminated structure during fabrication. This adhesive bonding between the piezoelectric active sensor and the structural substrate is the weak link in the sensory system because it deteriorates in time under environmental attacks (humidity, temperature cycles, etc.). There is an acute need for a better approach that would seamlessly connect the PWAS with the structure.

(2) Brittle PWAS can withstand very small bending. This brittleness of the PWAS imposes difficulties in handling and bonding of the sensor into the structure monitored.

(3) In addition, the conformability to curved surface is extremely poor so as to require extra treatment of the surfaces. ${ }^{[11][12][13]}$

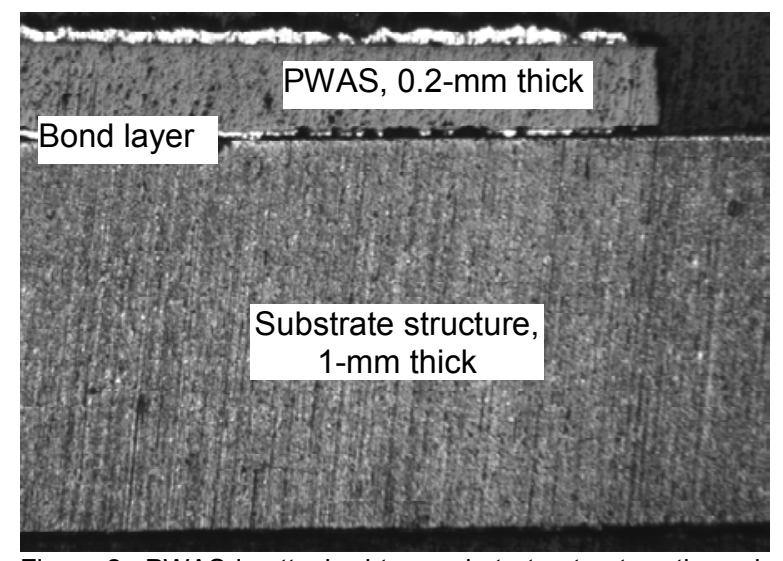

Figure 2 PWAS is attached to a substrate structure through the adhesive bond layer, which is susceptible to environmental attacks

The focus of our research was to create in-situ piezo active sensors using composite fabrication techniques. Composite PWAS are suitable for structural health monitoring due to the ease with which their mechanical properties may be tailored, low cost, ease of implementation, conformability to curved surface and compatibility with polymeric materials.

\section{STATE OF THE ART}

In recent years, several investigators have research the insertion of active material powders into composite materials to create active composites for actuator and sensing applications.

\section{Piezomagnetic Composite}

Terfenol-D is a magnetic anisotropy-compensated alloy $\mathrm{Tb}_{\mathrm{x}} \mathrm{Dy}_{1-\mathrm{x}} \mathrm{Fe}_{2}$ which shows a strong magnetostrictive behavior. When subjected to mechanical strain, Terfenol-D produces magnetic field. White ${ }^{[14]}$ reviewed the magnetostrictive tagging methodology of composites for structural health monitoring and measured the response of magnetostrictive-tagged composites under axial loading. Neat resin beams tagged $2.24 \%$ by volume with magnetostrictive Terfenol-D powder were used. Trovillion et $a l^{[15]}$ studied the magnetic characteristics of neat resin and glass-fiber-reinforced magnetostrictive composites subjected to axial load. The fiber reinforced polymer composite (FRP) beams consisted of 4 layers of continuous strand glass mat fibers embedded in a polyester resin. The top lamina of the composite was impregnated with Terfenol-D powder at a volume fraction of $2.24 \%$ for that lamina. The beams were subjected to uniaxial loading under load control at a rate of $0.02 \mathrm{kN} / \mathrm{s}$. Rearrangement of the magnetic dipoles chains of the magnetostrictive molecules through application of 
strong magnetic field was performed by applying a magnetic field through the thickness of the beam using a pair of magnets to apply a field of 800 Gauss.

Armstrong $^{[16]}$ presented a new model of nonlinear magneto-elastic behavior of magnetostrictive particulate composite. The analysis assumed uniform external magnetic field that is operating on a large number of well-distributed ellipsoidal magnetostrictive particles encased in a elastic, nonmagnetic composite matrix. Nersessian and Carman $^{[17]}$ studied five different volume-fraction of magnetostrictive particulate composites which were tested under two different conditions: (a) constant magnetic field and varying mechanical load; and (b) constant mechanical load and varying magnetic field. The results presented for the constant load indicated a strong dependence of strain output on applied prestress.

Krishnamurthy et $a .^{[18]}$ consider healthmonitoring detection of delaminations in composite materials using an excitation coil and a sensing coil. The open-circuit voltage induced in the sensing coil is proportional to the stress generated in the magnetostrictive layer by the presence of the delamination.

\section{Piezoelectric Composites}

The combination of polymer and piezoelectric ceramic to form composite PWAS offers the unique blending of the high electro-active properties of piezoelectric ceramics and the mechanical flexibility and formability of organic synthetic polymers. Recently, active composite PWAS have been developed, namely 1-3 composites by Smart Materials Corp, active fiber composite developed by MIT, and macrofiber composite (MFC) actuators at NASA Langley Center. These composite PWAS are capable of being repeatedly manufactured at low cost, are tolerant to damage, capable of conforming to complex or curved surfaces, and embeddable into structures. The uses of composite PWAS need to be investigated, and would provide the advantages of being robust, reliable, and easily adaptable for impedance-based health monitoring.

Composites PWAS can be classified according to the connectivity of piezoelectric ceramics and matrix phases. The composite PWAS in this paper has a 0-3 connectivity pattern. The " $0-3$ " means that the ceramic particles are randomly dispersed in a polymer matrix. 0-3 composites can be more easily fabricated in complex shapes than other forms of composites. Various approaches have been tried for producing 0-3 composites. ${ }^{[13][19][20]}$

\section{SELF-SENSING PIEZOMAGNETIC COMPOSITES EXPERIMENTS}

An piezomagnetic composite beam was fabricated by combining 7 layers of a woven glass roving 36oz./sq.yd with Atlac 580-05 Urethane-modified Vinyl Ester resin. The beam was $1000 \mathrm{~mm}$ long, and had a $100 \mathrm{~mm}$ by $6.5 \mathrm{~mm}$ cross section. Terfenol-D tagging powder was used in the two outer laminae in the middle $500 \mathrm{~mm}$ of the span. Of the 1000 grams of resin used, 250 grams had the MS powder. The resin was cured with $1 \%$ MEKP (methyl ethyl ketone peroxide) at room temperature for approximately 90 minutes. The target weight fraction of the glass fibers in the composite was $\mathrm{w}_{\mathrm{f}}=30 \%$. The weight fraction of MS Terfenol-D tagging was $15 \%$.

The beam was instrumented with strain gages in the mid-span section on the upper and lower surfaces. The magnetic flux density was measured with the gaussmeter. A special fixture was constructed to ensure consistent alignment of the gaussmeter probe with the composite surface and to mechanically protect it. The tip of the gaussmeter probe is made of ceramic and covers the magnetic sensor. On advice from the gaussmeter manufacturer (Lakeshore Cryotonics, Inc.) a protective wood fixture shown in Figure 8 was designed and fabricated. The distance from the tip of the sensor to the upper surface of the beam was $2 \mathrm{~mm}$. The experimental setup is shown in Figure 3.

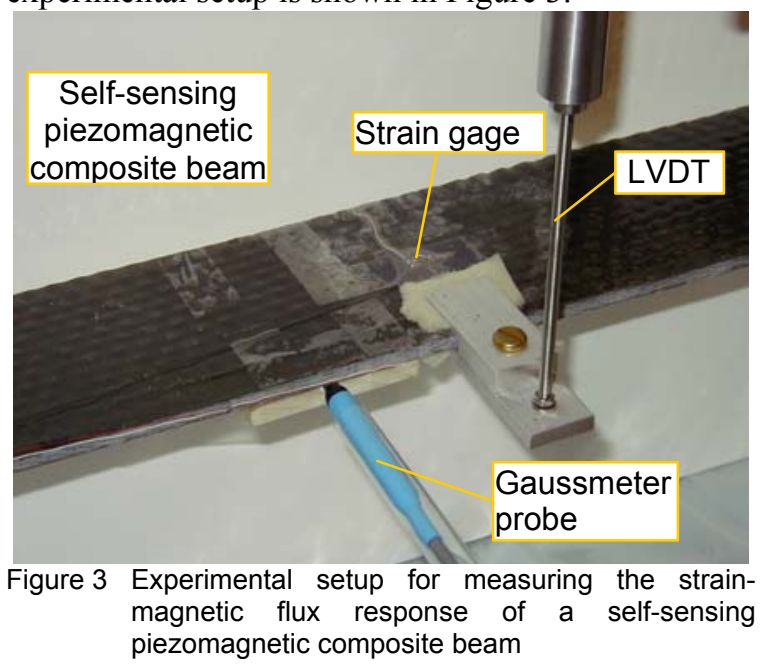

The magnetic flux density response of the beam is presented was measured for ten loading-unloading cycles. The zero load reading was substracted from the other values in order to eliminate the influence of the environmental magnetic field. The relationship between magnetic flux density and strain is presented in Figure 4. The ten cycles considered in the experiment showed the reproducibility of the magnetic flux readings. The relationship between 
strain and magnetic flux density was consistent and repeatable. Also noticed was that the magnetic flux had a small hysteresis. This phenomenon is present because of changes in the microscopic magnetic status of the piezomagnetic particles. The small difference between readings in different cycles illustrates the deviation expected from the use of general-purpose magnetic flux density measurement equipment.

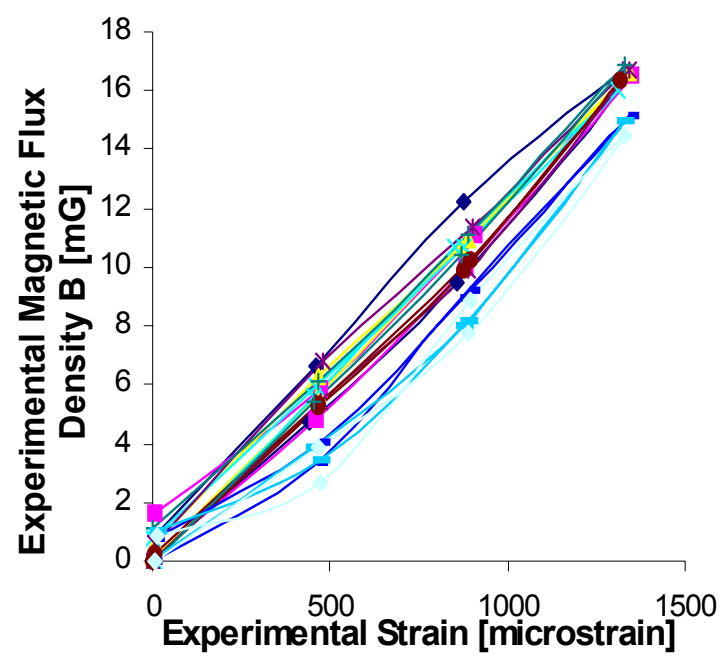

Figure 4 Magnetic flux response with strain of a piezomagnetic composite beam

\section{PIEZOELECTRIC COMPOSITES}

Another approach considered in our research was that of piezoelectric composites. The piezoelectric composites have the advantage that electric signals can be collected directly, whereas in the case of piezomagnetic composites magnetic flux probes had to be used. Our purpose in pursuing the fabrication of piezoelectric composites was to eventually be able to achieve in-situ fabrication of PWAS of a quality comparable with that of ceramic PWAS. By fabricating the PWAS directly onto the structure a seamless bond would be achieved between the PWAS and the structure that would be impervious to environmental attacks can be created. Thus, we will eliminate the "weak link" in the present use of bonded PWAS and achieve a long time durability of the embedded sensory system.

\section{PREPARATION OF PIEZOELECTRIC COMPOSITE PWAS PREPARATION}

The epoxy resin used in the fabrication of the piezoelectric composite PWAS was EPO-TEK 301-2 from Epoxy Technology, Inc.. This epoxy has a good handling property, high dielectric strength and a low viscosity. The mixture ratio for the epoxy part " $A$ " and part " $\mathrm{B}$ " (hardener) is specified as 1: 0.35 by the supplier and was followed in this study.

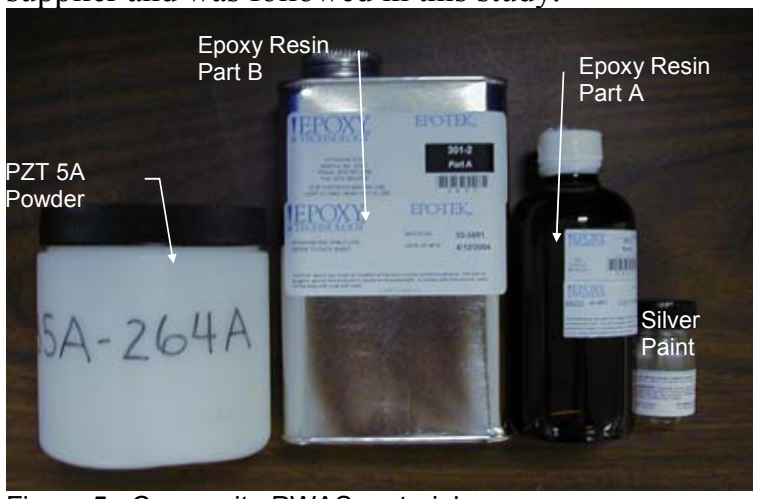

Figure 5 Composite PWAS materials

The PZT particles in the composite PWAS were PZT-5B powders from Morgan Electro Ceramics. PZT-5B powders have a high sensitivity and high time stability (Figure 5).

The silver paint used for creating the electrodes was acquired from SPI Supplies and Structure Probe, Inc.

The poling workstation consists of an oven, a constant DC power supply, a high voltage power supply, a multimeter and a thermometer (Figure 6).

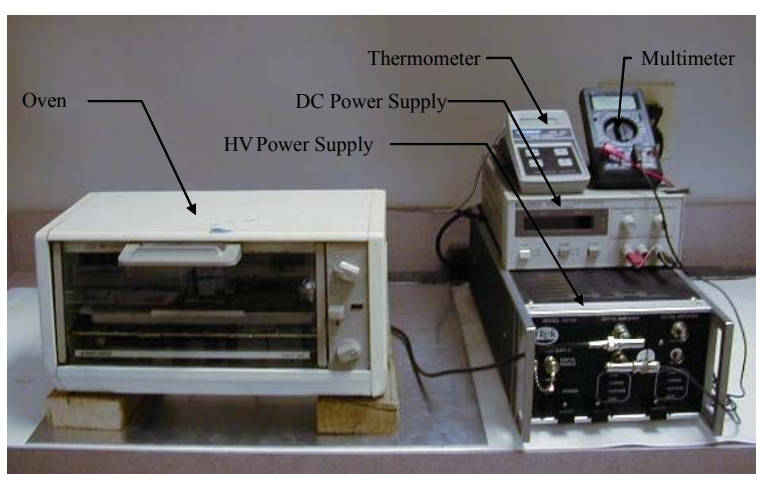

Figure 6 Poling Work Station

\section{PIEZOELECTRIC COMPOSITE PWAS FABRICATION}

Clean the surface of host structure and put a mask. The mask has a $7 \mathrm{~mm}$ diameter, $0.2 \mathrm{~mm}$ thickness hole and its center is in the desired position. PZT powder with a weight fraction of $85 \%$ was added to the epoxy matrix phase and was stirred thoroughly. The paste was then spread into the mask and let it cure at an elevated temperature (50 degree C) until hard. Remove excess and sand it down to final thickness. A small piece of copper foil was applied to one side of the cured composite PWAS to form an electrode. After curing and electroding had 
been completed, poling of the composite PWAS was subsequently carried out to activate its piezoelectric effect. A constant DC voltage of $1 \mathrm{kV}$ was applied to the composite PWAS to pole the sample for four hours at 80 degree $\mathrm{C}$.

\section{CHARACTERIZATION OF PIEZOELECTRIC COMPOSITE PWAS}

We measured the final size of the composite PWAS. It is $7 \mathrm{~mm}$ diameter and $0.2 \mathrm{~mm}$ thickness which is same as the traditional PWAS. The electricity of the composite PWAS was measured before and after poling. The resistance is greater than $40 \mathrm{M} \Omega$ before and after poling. The resistivity is higher than $40 \mathrm{M} \Omega \mathrm{m}$. The capacitance of composite is only $0.02 \mathrm{nF}$ which is lower than the traditional PWAS value $3 \mathrm{nF}$.

\section{IMPACT TEST ON PIEZOELECTRIC COMPOSITE PWAS}

The effectiveness of the composite PWAS for impact was investigated based on an impact test setup show in Figure 7.

Impact point showed in the Figure 7 and it is $20 \mathrm{~mm}$ away from the center of the composite PWAS. The output of the composite PWAS was measured simply as a voltage signal using a digital oscilloscope.

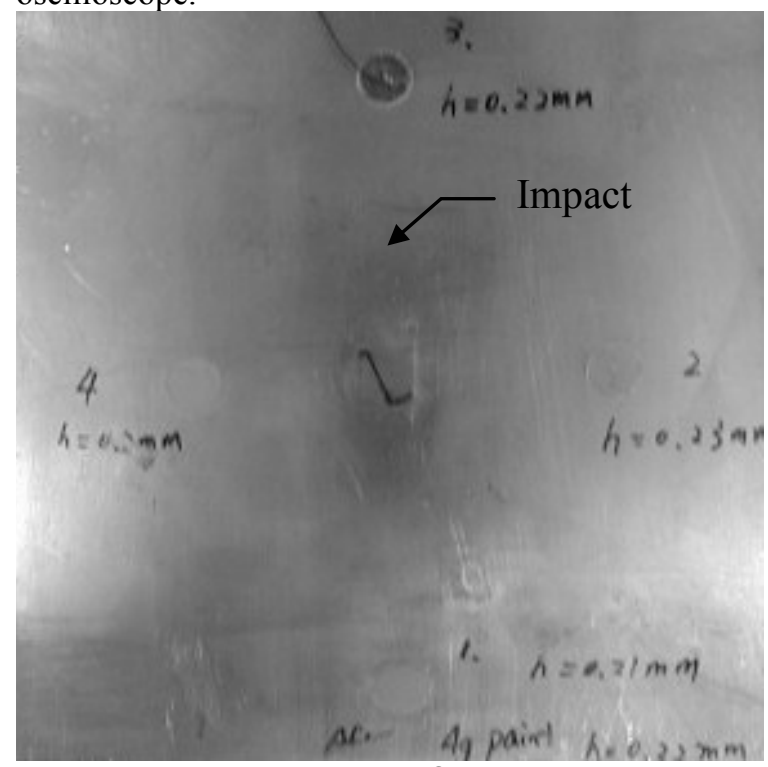

Figure 7 Impact composite PWAS sample
We measured the voltage signal before and after poling. Before poling, the voltage signal damped out very soon. After poling, we can find the voltage amplitude is much higher than that before poling. And it also shows some vibration signal after the impact. From this figure, it is seen that upon hit, the beam vibrated first and then slowly damped out. The composite PWAS have a good repeatability in its output signal when subjected to similar impact forces.
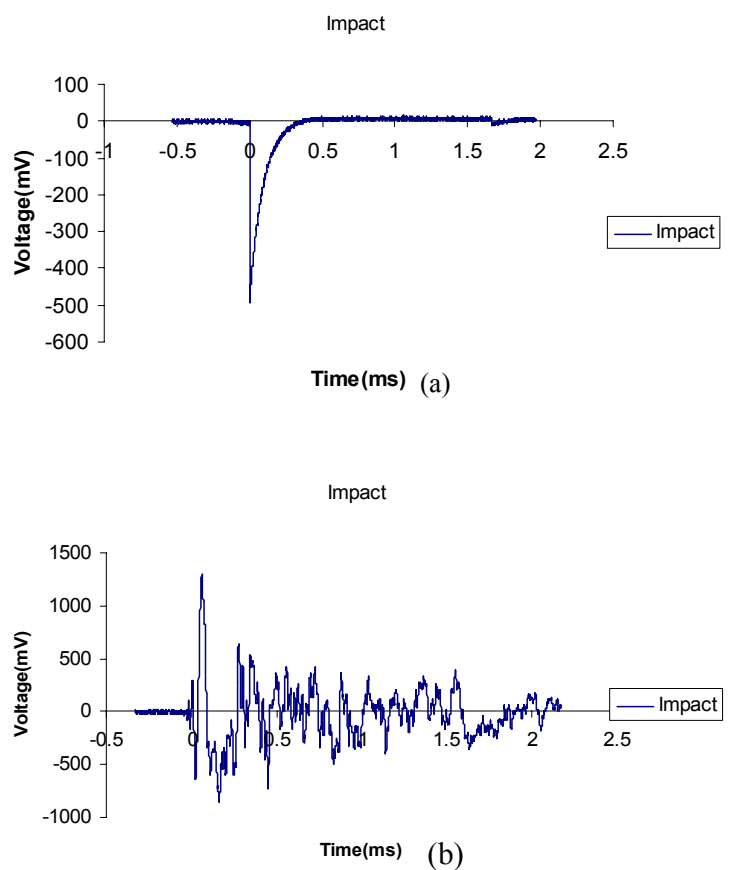

Figure 8 The electric voltage signal measured by the impact hammer, (a) before poling (b) after poling

\section{PIEZOELECTRIC COMPOSITE PWAS VIBRATION TEST}

The effectiveness of the composite PWAS for dynamic measurement was investigated based on a vibration test setup show in Figure 9. The beam was mounted as a cantilever in a calibrated cantilever fixture. A composite PWAS was placed on the top of the beam and a traditional PWAS was place at the other side of the beam. The tip of the beam was displaced to a certain value (approx. $10 \mathrm{~mm}$ ) and then suddenly released such as the beam entered in free vibration. 
(a)

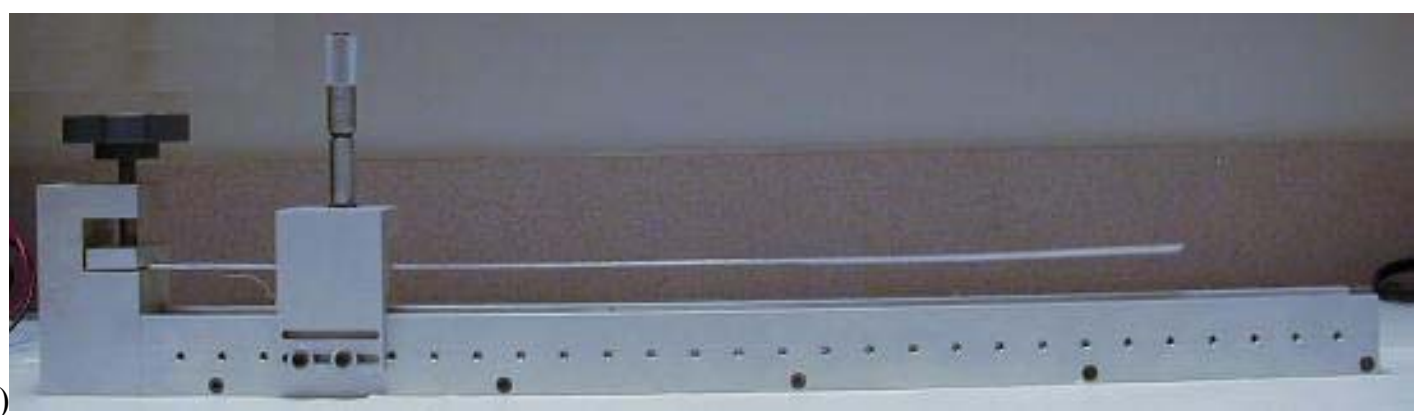

(b)
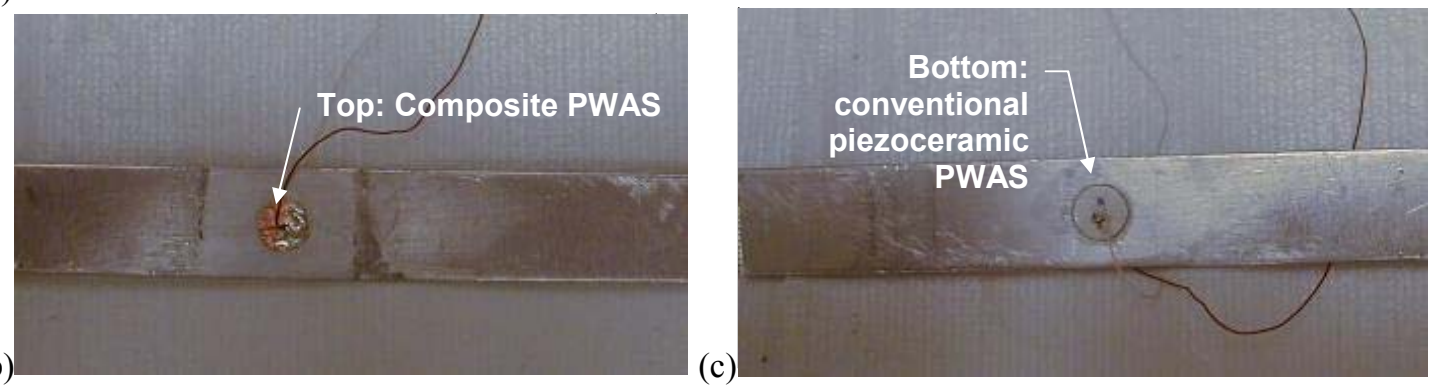

Figure 9 Piezoelectric composite PWAS fabricated on a cantilever beam: (a) experimental setup; (b) close-up view of the top surface showing the piezoelectric composite PWAS; (c) close-up view of the bottom surface showing the conventional piezoceramic PWAS

An oscilloscope connected directly to the PWAS was used to record the electric signals induced in both PWAS by the piezoelectric coupling between the mechanical vibration and the electric field (Figure 10). In Figure 10 (a), it is traditional PWAS voltage signal. We can easily see it reflected the beam vibration very well. But before and after poling, we still can't find the signal from the composite PWAS. Figure 10 (b) only showed the noise.

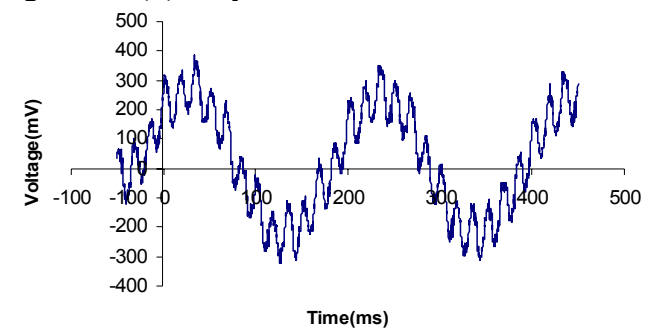

(a)

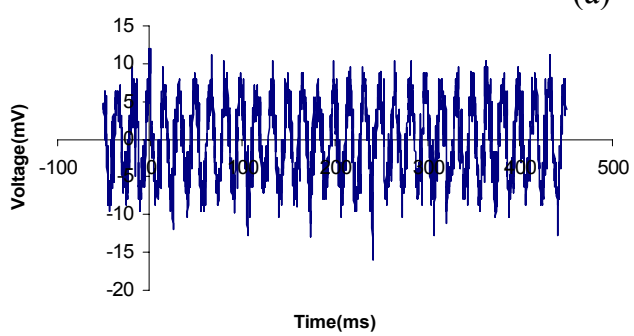

(b)

Figure 10 Measured electric signals during the plucked beam experiments: (a) conventional piezoceramic PWAS; (b) piezoelectric composite PWAS

\section{CONCLUSIONS}

A technique for fabricating composite PWAS has been established. The effectiveness of composite PWAS has been preliminarily characterized through a series of tests, which include impact test and vibration test. The experiment results of the composite PWAS have been compared with the traditional PWAS. The experimental results in this study gave the basic demonstration of the piezoelectricity of composite PWAS. The composite PWAS are capable of use as an active sensor.

Our future work is focused on perfecting the composite technology methods for the in-situ fabrication of composite PWAS. The composite fabrication methods considered for investigation are: (a) mask; (b) poling; (c) powders technology; (d) coatings. The ultimate vision is to create a methodology to create the composite PWAS directly onto the structural substrate through a easy technique followed by electroding and poling.

\section{ACKNOWLEDGMENT}

Support from the Air Force Research Lab through UTC contract \#03-S470-033-C1 of F33615-01-D5801 is thankfully acknowledged.

\section{REFERENCES}

[1] Keilers, C. H.; Chang, F.-K. (1995) "Identifying 
Delamination in Composite Beam using Built-in Piezoelectrics", Journal of Intelligent Material Systems and Structures, Vol. 6, 1995, pp. 647672

[2] Chang, F.-K. (1995) "Built-In Damage Diagnostics for Composite Structures", in Proceedings of the $10^{\text {th }}$ International Conference on Composite Structures (ICCM-10), Vol. 5, Whistler, B. C., Canada, August 14-18, 1995, pp.283-289

[3] Chang, F.-K. (1998) "Manufacturing and Design of Built-in Diagnostics for Composite Structures", $52^{\text {nd }}$ Meeting of the Society for Machinery Failure Prevention Technology, Virginia Beach, VA, March 30 - April 3, 1998.

[4] Wang, C. S.; Chang, F.-K. (2000) "Built-In Diagnostics for Impact Damage Identification of Composite Structures", in Structural Health Monitoring 2000, Fu-Kuo Chang (Ed.), Technomic, 2000, pp. 612-621

[5] Lin, X.; Yuan, F. G. (2001a) "Diagnostic Lamb Waves in an Integrated Piezoelectric Sensor/Actuator Plate: Analytical and Experimental Studies", Smart Materials and Structures, Vol. 10, 2001, pp. 907-913

[6] Lin, X; Yuan, F. G. (2001b) "Damage Detection of a Plate using Migration Technique", Journal of Intelligent Material Systems and Structures, Vol. 12, No. 7, July 2001

[7] Ihn, J.-B.; Chang, F.-K. (2002) "Built-in diagnostics for monitoring crack growth in aircraft structures", Proceedings of the SPIE's $9^{\text {th }}$ International Symposium on Smart Structures and Materials, 17-21 March 2002, San Diego, CA, paper \#4702-04

[8] Giurgiutiu, V.; Zagrai, A. (2000) "Characterization of Piezoelectric Wafer Active Sensors", Journal of Intelligent Material Systems and Structures, Sage Pub., UK, Vol. 11, No. 12, December 2000, pp. 959-976

[9] Giurgiutiu, V.; Zagrai, A. N.; Bao J.; Redmond, J.; Roach, D.; Rackow, K. (2002) "Active Sensors for Health Monitoring of Aging Aerospace Structures", International Journal of the Condition Monitoring and Diagnostic Engineering Management, UK, Vol. 5, No. 3, August 2002

[10] Giurgiutiu, V.; Bao, J.; Zhao, W. (2003) "Piezoelectric-Wafer Active-Sensor Embedded Ultrasonics in Beams and Plates", Experimental Mechanics, Sage Pub. December 2003, pp. 428449

[11] Pomirleanu, R.; Giurgiutiu, V. (2003) "Full-Power Dynamic Characterization of Piezoelectric and Magnetostrictive Actuators", Journal of Intelligent Material Systems and Structures, Sage Pub. (in press)

[12] Giurgiutiu, V.; Zagrai, A.; Bao, J. (2004) "Damage Identification in Aging Aircraft Structures with Piezoelectric Wafer Active Sensors", Journal of Intelligent Material Systems and Structures, Sage Pub., Vol. 15, No. 6, June 2004 (in press)

[13] Zhang, Y., "Dynamic Strain Measurement Using
Piezoelectric Paint", 4th International Workshop on Structural Health Monitoring, September 1517, 2003, Stanford University, CA, pp. 1446-1452

[14] White, S. R. (1999) "Magnetostrictive Tagging of Composites for Health Monitoring", Proceedings, 1999 International Composites Expo, Cincinnati, $\mathrm{OH}$, May 10-12, 1999, pp. 22E1 - 22E6.

[15] Trovillion, J. Kamphaus J., Quattrone R., Berman J. (1999) "Structural Integrity Monitoring Using Smart Magnetostrictive Composites", Proceedings, International Composites EXPO'99, Cincinnati, $\mathrm{OH}, 1999$, session 22-D, pp. 1-6

[16] Armstrong, W. D. (2000) “ A General MagnetoElastic Model of Terfenol-D Particle Actuated Composite Material", Adaptive Structures and Materials 2000, ASME International Mechanical Engineering Congress and Exposition, Nov.5-10, 2000, Orlando, Florida, pp 127-137

[17] Nersessian, N., Carman, G. (2000) “ Magnetomechanical Characterization of Magnetostrictive Composite", Adaptive Structures and Materials 2000, ASME International Mechanical Engineering Congress and Exposition, Nov.5-10, 2000, Orlando, Florida, pp 139-145

[18] Krishnamurthy, A. V.; Anjanappa, M.; Wang, Z. and Chen, Z. (1999) " Sensing of Delaminations in Composite Laminates using Embedded Magnetostrictive Particle Layers", Journal of Intelligent Material Systems and Structures, Vol.10 October 1999

[19] Egusa, S., Iwasawa. " Piezoelectric paints: preparation and application as built-in vibration sensors of structural materials," J. Material Science, 28: 1667-1672, 1993

[20] Egusa, S., N. Iwasawa., "Piezoelectric paints as one approach to smart structural materials with health-monitoring capabilities," Smart Mater. Struct., 7: 438-445: 1998 